\title{
VARIABLE SPECTRA OF IC 4997 AND NGC 6572
}

\author{
SIEK HYUNG and LAWRENCE H. ALLER \\ University of California, 8979 Math Science bldg., 405 Hilgard Ave. \\ Los Angeles, California 90024-1562 \\ and \\ WALTER A. FEIBELMAN \\ NASA/Goddard Space Flight Center, Code 684, Greenbelt, Maryland 2077
}

Variability of the [OIII] $4363 / 4340 \mathrm{H} \gamma$ ratio in IC 4007 was established in 1956 by William Liller and L.H. Aller who attributed the changes to a gradual decrease of electron density with time. Subsequent $4363 / 4340$ ratio fluctuations negated this explanation. Ferland pointed out that small changes in the radiative flux of the Planetary nebula nucleus (PNN) could explains the variations. Our pervious study emphasized IUE observations, here we compare high dispersion spectra obtained with the Hamilton Echelle Spectrograph with previous measurements to asses line intensity variations. Emission line variability in PNN spectra as noted by Mendez et al. (1988) and by other for HeII 4686 in NGC 6572 may offer significant clues. PNN 4686 appeared by 1990 in IC 4997. Possibly both of these PNN may be evolving into Wolf-Rayet objects, but this development does not necessarily imply that the nebular excitation will increase with time.

Attempts to interpret these nebulae with the aid of theoretical models encountered troubles. Many trials for IC 4997 resulted in a model consisting of a dense inner shell $\left(N_{H} \sim 10^{7}\right.$ atoms $\left./ \mathrm{cm}^{3}\right)$ of radius $0.0005 \mathrm{pc}$ and outer radius 0.000565 pc surrounded by a shell of lower density $\left(N_{H} \sim 10,000\right.$ atoms $\left./ \mathrm{cm}^{3}\right)$ with an outer radius of $0.013 \mathrm{pc}$. This model, however, encounters a paradox in that with an expansion velocity of $20 \mathrm{~km} / \mathrm{sec}$, the inner shell would have left the photosphere of the PNN about 30 years ago. These is no evidence for any unusual events in the star and nebula at that time. If a greater distance is assumed for IC 4997, the date of ejection can be pushed backward but then difficulties are encountered if we try to reproduce the observed nebular line intensities.

For NGC 6572 a toroid of density $N_{H}=25,000$ atoms $/ \mathrm{cm}^{3}$, inner radius of $0.01 \mathrm{pc}$ and thickness $\sim 0.014 \mathrm{pc}$ was combined with a conical segment of density $N_{H}=10,000$ atoms $/ \mathrm{cm}^{3}$, inner radius $0.04 \mathrm{pc}$, and thickness $0.0102 \mathrm{pc}$. T(PNN)= 55,000 and 50,000 for IC 4997 and NGC 6572 respectively. While we cannot assert that long term changes are taking place in either of these PNs, their continued monitoring to check on spectral variations is recommended. Gurzadyan has suggested that the [OIII] $4363 / \mathrm{H} \gamma$ ratio varies with a 50 year cycle upon which smaller fluctuations are superposed, but the most recent (1992) ratio measurements do not seem to support this conclusion. 